\title{
Determining the desert dust aerosol presence in the Mantaro Valley, Peru
}

\section{Determinación de la presencia de aerosoles de polvo desértico en el Valle del Mantaro, Perú}

\author{
I. Y. Platero ${ }^{1 *}$, R. Estevan ${ }^{1,2}$, A. Moya ${ }^{2}$, R. A. Yuli ${ }^{3}$ \\ 1. Universidad Continental, Huancayo, Perú \\ 2. Instituto Geofísico del Perú, Lima, Perú \\ 3. Universidad Nacional Mayor de San Marcos, Lima, Perú \\ ${ }^{(*)}$ E-mail: nenagianna11@gmail.com \\ Received: 11/01/2018 Accepted: 21/06/2018 \\ DOI: DOI: $10.7149 /$ OPA.51.3.50023
}

\begin{abstract}
:
Since March 19, 2015, a sunphotometer CIMEL CE-318T has been installed in the Huancayo Observatory, belonging to the Geophysical Institute of Peru. Aerosol Optical Depth at $440 \mathrm{~nm}\left(\tau_{440}\right)$ and the Ångström Exponent $(\alpha)$ derived from columnar aerosol sun-photometer measurement, based on Aerosol Robotic Network (AERONET) products, has been used for aerosols classification. The used AERONET dataset correspond to quality Level 2.0. A reliable identification of desert dust events has been possible based on above mentioned parameters. A total of 5 days and 10 hours have been identified as desert dust events based on AERONET dataset and the air mass backtrajectories employing the Hybrid Single Particle Lagrangian Integrated Trajectory Model (HYSPLIT). Simulations carried out with the Weather Research and Forecasting (WRF) Model has been used to corroborate the desert dust events. The most intense event was recorded in December 27, 2016, with a maximum AOD value of 0.32 at 12:03 GMT. It has been shown that the major contribution to AOD on days identified as desert dust events, corresponds to desert dust aerosols. This is the first report for Peru, of desert dust aerosols transport from the Peruvian coastal desert to the central Andes, specifically to the Mantaro Valley.
\end{abstract}

Key words: AOD; sunphotometer; AERONET; desert dust

\section{RESUMEN:}

Desde el 19 de marzo de 2015, se ha instalado un fotómetro solar del tipo CIMEL CE-318T en el Observatorio de Huancayo, perteneciente al Instituto Geofísico del Perú. El Espesor Óptico por Aerosoles a $440 \mathrm{~nm}\left(\tau_{440}\right)$ y el Exponente de Ångström $(\alpha)$ derivados de las mediciones columnares del fotómetro solar, basados en los productos de la Red Robótica de Aerosoles (AERONET), se han utilizado para la clasificación de los aerosoles. El conjunto de datos de utilizados corresponde al nivel de calidad 2.0 de AERONET. La identificación confiable de los eventos de polvo desértico ha sido posible en base a los parámetros mencionados anteriormente. Se identificaron un total de 5 días y 10 horarios como eventos de polvo desértico, basados en los datos de AERONET y las retrotrayectorias de masas de aire empleando el Modelo de Trayectoria Integrada Lagrangiana de Partícula Híbrida (HYSPLIT). Las simulaciones llevadas a cabo con el modelo de investigación y pronóstico del tiempo (WRF) se ha utilizado para corroborar los eventos de polvo desértico. El evento más intenso se registró el 27 de diciembre de 2016, con un valor máximo de AOD de 0.32 a las 12:03 GMT. Se ha evidenciado que la principal contribución al EOA en los días identificados como eventos de polvo desértico corresponde a los aerosoles de polvo desérticos. Este es el primer reporte para Perú, sobre el transporte de aerosoles de polvo desértico desde el desierto costero peruano hasta los Andes centrales, específicamente al Valle del Mantaro.

Palabras clave: AOD; fotómetro solar; AERONET; polvo desértico 


\section{REFERENCES AND LINKS / REFERENCIAS Y ENLACES}

[1] UNEP, WMO, UNCCD, Global Assessment of Sand and Dust Storms. United Nations Environment Programme, Nairobi, (2016).

[2] F. Briceño-Zuluaga, A. Castagna, J. A. Rutllant, V. Flores-Aqueveque, S. Caquineau, A. Sifeddine, F. Velazco, D. Gutierrez, J. Cardich, "Paracas dust storms: Sources, trajectories and associated meteorological conditions", Atmospheric Environment 165, 99-110 (2017).

[3] R. Estevan, L. Mona, N. Papagiannopoulos, J.C. Antuña, V. Cachorro, Á. de Frutos, "CALIPSO and sunphotometer measurements of Saharan dust events over Camagüey," Opt. Pura Apl. 47 (3) 189-196 (2014).

[4] C. Velasco-Merino, D. Mateos, C. Toledano, J. M. Prospero, J. Molinie, L. Euphrasie-Clotilde, R. González, V. E. Cachorro, A. Calle, A. M. de Frutos, "Impact of long-range transport over the Atlantic Ocean on Saharan dust optical and microphysical properties," Atmos. Chem. Phys. Discuss. in review (2017).

[5] WHO, Review of evidence on health aspects of air pollution. REVIHAAP Project, Technical Report; WHO Regional Office for Europe, Scherfigsvej 8, DK-2100 Copenhagen Ø, Denmark; 309 pp (2013).

[6] M. C. Fisher, G. L. Koenig, T. White, J. Taylor, "Pathogenic clones versus environmentally driven population increase: analysis of an epidemic of the human fungal pathogen Coccidiodes immitis". Journal of Clinical Microbiology 38, 807-813 (2000).

[7] J. Boreson, A. M. Diller, J. Peccia, Correlating bioaerosol load with PM2.5 and PM10cf concentrations: a comparison between natural desert and urban-fringe aerosols. Department of Civil and Environmental Engineering, Engineering Center and Department of Chemical and Materials Engineering, Arizona State University, Tempe, AZ (2004).

[8] R. Washington, G.F.S. Wiggs, Desert dust. In Arid Zone Geomorphology: Process, Form and Change in Drylands (ed. Thomas, D.S.G.) pp. 517-538. 3rd Edition. Wiley (2011).

[9] A. S. Goudie, "Dust storms: recent developments". Journal of Environmental Management 90, 89-94 (2009).

[10] P. Knippertz, Meteorological aspects of dust storms. In Mineral Dust: A key Player in the Earth System (ed. Knippertz, P. and Stuut, J.B.W.). pp.121-147. Springer (2014).

[11] A. T. Evan, J. Dunion, J. A. Foley, A. K. Heidinger, C. S. Velden, "New evidence for the relationship between Atlantic tropical cyclone activity and African dust outbreaks," Geophysical Research Letters 33, L19813 (2006).

[12] A. Nenes, B. Murray, A. Bougiatioti, Mineral dust in microphysical interactions with clouds. In Mineral Dust: A key Player in the Earth System (ed. Knippertz, P. and Stuut, J-B.W.). pp.287-325. Springer (2014).

[13] D. F. Escobar, Evaluación climatológica y sinóptica del fenómeno de vientos Paracas. Lima, Perú. Tesis para optar el título de Ingeniero Meteorológico, Universidad Nacional Agraria La Molina, 62 pp (1993). https://es.scribd.com/doc/8839005/

[14] J. J. Quijano, Estudio numérico y observacional de la dinámica de Viento Paracas, asociado al transporte eólico hacia el océano frente a la costa de Ica-Perú. Lima - Perú: Universidad Peruana Cayetano Heredia. MSc Thesis, 151p. (2013). http://www.met.igp.gob.pe/publicaciones/2013/JQuijano_tesisMSc.pdf

[15] B. N. Holben, T. F. Eck, I. Slutsker, D. Tanré, J. P. Buis, A. Setzer, E. F. Vermote, J. A. Reagan, Y. J. Kaufman, T. Nakajima, F. Lavenu, I. Jankowiak, A. Smirnov, “AERONET - A federated instrument network and data archive for aerosol characterization," Remote Sens. Environ. 66, 1-16 (1998).

[16] B. N. Holben, D. Tanré, A. Smirnov, T. F. Eck, I. Slutsker, N. Abuhassan, W. W. Newcomb, J. S. Schafer, B. Chatenet, F. Lavenu, Y. J. Kaufman, J. Vande Castle, A. Setzer, B. Markham, D. Clark, R. Frouin, R. Halthore, A. Karneli, N. T. O'Neill, C. Pietras, R. T. Pinker, K. Voss, G. Zibordi, “An emerging groundbased aerosol climatology: Aerosol optical depth from AERONET", J. Geophys. Res. 106, 12067-12097 (2001).

[17] G. A. D’Almeida, P. Koepke, E. P. Shettle, Atmospheric Aerosol: Global Climatology and Radiative Characteristics. Hampton, Virginia: A Deepak Publishing Co., (1991).

[18] R. Vergaz, V. E. Cachorro, A. M. de Frutos, J. M. Vilaplana, B. A. de la Morena. "Columnar characteristics of aerosols by spectroradiometer measurements in the maritime area of the Cadiz Gulf (Spain)," Int. J. Climatol 25, 1781-1804 (2005). 
[19] S. Verma, D. Prakash, P. Ricaud, S. Payra, J. L. Attié, M. Soni, "A New Classification of Aerosol Sources and Types as Measured over Jaipur, India." Aerosol and Air Quality Research 15, 985-993 (2015).

[20] C. Toledano, V. E. Cachorro, A. M. de Frutos, M. Sorribas, N. Prats, B. A. de la Morena, "Inventory of African desert dust events over the southwestern Iberian Peninsula in 2000-2005 with an AERONET Cimel Sun photometer," J. Geophys. Res., 112, D21201, (2007).

[21] V. E. Cachorro, M. A. Burgos, D. Mateos, C. Toledano, Y. Bennouna, B. Torres, A. M. de Frutos, A. Herguedas, "Inventory of African desert dust events in the north-central Iberian Peninsula in 20032014 based on sun-photometer-AERONET and particulate-mass-EMEP data," Atmos. Chem. Phys., 16, 8227-8248 (2016).

[22] R. R. Draxler, G. D. Rolph, HYSPLIT (Hybrid Single-Particle Trajectory), model access via NOAA ARL READY Website (http://www.arl.noass.gov/hysplit4.html). NOAA Air Resources Laboratory, Silver Spring, MD (2003).

[23] C. Aybar, W. Lavado-Casimiro, A. Huerta, C. Fernández, F. Vega, E. Sabino, O. Felipe-Obando, Uso del Producto Grillado "PISCO" de precipitación en Estudios, Investigaciones y Sistemas Operacionales de Monitoreo y Pronóstico Hidrometeorológico. Nota Técnica 001 SENAMHI-DHI-2017, Lima-Perú (2017).

[24] M. A. Burgos, D. Mateos, V. E. Cachorro, C. Toledano, A. M. de Frutos, "Aerosol properties of mineral dust and its mixtures in a regional background of north-central Iberian Peninsula," Sci Total Environ 572, 1005-1019 (2016).

[25] N. T. O'Neill, Dubovik, O., Eck, T. F., "A modified Angstrom coefficient for the characterization of submicron aerosols", App. Opt., 40 (15), 2368-2374 (2001).

[26] N. T. O'Neill, T. F., Eck, A. Smirnov, B. N.Holben, S. Thulasiraman, "Spectral discrimination of coarse and fine mode optical depth", 108 (D17), 4559-4573, 10.1029/2002JD002975, (2003).

[27] D. Gutiérrez, I. Bouloubassi, A. Sifeddine, S. Purca, K. Goubanova, M. Graco, D. Field, L. Méjanelle, F. Velazco, A. Lorre, R. Salvatteci, D. Quispe, G. Vargas, B. Dewitte, L. Ortlieb, "Coastal cooling and increased productivity in the main upwelling zone off Peru since the mid-twentieth century". Geophys. Res. Lett. 38, 1-6 (2011).

[28] W. J. Sydeman, M. García-Reyes, D. S. Schoeman, R. R. Rykaczewski, S. a Thompson, B. a Black, S. J. Bograd, "Climate change and wind intensification in coastal upwelling ecosystems," Sci. New York 345, 77-80 (2014).

\section{Introducción}

El estudio de los aerosoles atmosféricos en general y los de origen natural, en particular, cobra cada vez mayor interés en la comunidad científica internacional debido a los efectos directos e indirectos que estos tienen sobre los fenómenos relacionados con la interacción atmósfera-océano, la calidad del aire y la salud humana [1]. Los aerosoles de origen desértico y el polvo mineral, constituyen un tipo común de material particulado en suspensión, que caracteriza las regiones áridas del planeta y cuyo principal mecanismo de generación es la erosión debido al viento [2]. Estos aerosoles pueden ser transportados a enormes distancias desde su región de origen, como es el caso de los aerosoles desérticos que se originan en el Sahara y alcanzan el Caribe [3] y afectar las condiciones del tiempo y el clima en zonas ubicadas a grandes distancias de la fuente donde se han generado [4]. El polvo mineral en suspensión es una de las fuentes de especies alérgicas potenciales, constituyendo uno de los problemas que agrava la salud humana [5], como la alergia de tipo estacional y enfermedades respiratorias, las cuales pueden coincidir con elevadas concentraciones de polvo incluyendo el desértico [6,7].

Las partículas de polvo afectan los procesos y sistemas relacionados con la atmósfera, así como, los océanos y la litósfera [8] donde provoca la fertilización tanto de los mares como de los bosques [9]. Las interacciones del polvo con el medio ambiente son muy complejas y aún no son comprendidas en su totalidad [10]. El efecto del polvo en el sistema climático se produce mediante la modificación de la intensidad de las tormentas tropicales y los tiempos de vida de los huracanes y sus intensidades [11]. La influencia del polvo en la modificación de balance radiativo de la tierra puede tener efectos en el ciclo hidrológico de la tierra favoreciendo, en algunos casos, la precipitación, al servir como núcleos de condensación [12]

La existencia de extensas regiones desérticas en el Perú ha motivado el estudio de la dinámica de los vientos y de los mecanismos que generan el levantamiento de polvo en estas zonas [2]. Sin embargo, aún son escasos los trabajos que aborden esta temática y los que existen se concentran fundamentalmente en la región de 
Paracas y el estudio del viento homónimo [13,14]. No se han encontrado evidencias de estudios previos sobre el transporte de aerosoles de polvo desértico, desde alguno de los desiertos costeros del Perú, hacia los Andes Centrales, lo cual ha servido como motivación para este trabajo. Sin embargo, es preciso tener en cuenta que este estudio constituye una primera aproximación sobre estos eventos, a partir de las mediciones de fotometría solar disponibles en el Observatorio de Huancayo (OHY). Dada la dificultad que implica la determinación del origen desértico de los aerosoles, a partir de las mediciones antes mencionadas, debido entre otros aspectos a la posible mezcla de aerosoles y a la corta duración de estos episodios, sería propicio, en un futuro, contar con otro tipo de mediciones que permitieran caracterizar, por ejemplo, la composición química de los aerosoles registrados.

\section{Materiales y Métodos}

El 19 de marzo de 2015 fue instalado en el Observatorio de Huancayo (12.040 S, 75.32090 0, 3352.0 msnmm), perteneciente al Instituto Geofísico del Perú, un fotómetro solar del tipo CIMEL CE-318T, también conocido como fotómetro lunar. Este instrumento forma parte de la Red Robótica de Aerosoles (AERONET) $[15,16]$. Para este estudio se emplearon los datos de Espesor Óptico por Aerosoles en la longitud de onda de $440 \mathrm{~nm}\left(\tau_{440}\right)$, y el Parámetro de Angstrom $(\alpha)$ en el rango de longitudes de onda de $440-870 \mathrm{~nm}$, registrados en el OHY, para el período comprendido entre el 19 de marzo de 2016 y el 31 de agosto de 2017. Para determinar la presencia de aerosoles desérticos en el OHY es preciso, primeramente, seleccionar los casos que correspondan a aerosoles del tipo polvo. Con este propósito se elaboró un diagrama de dispersión entre $\tau_{440} \mathrm{y} \alpha$ (Fig. 1).

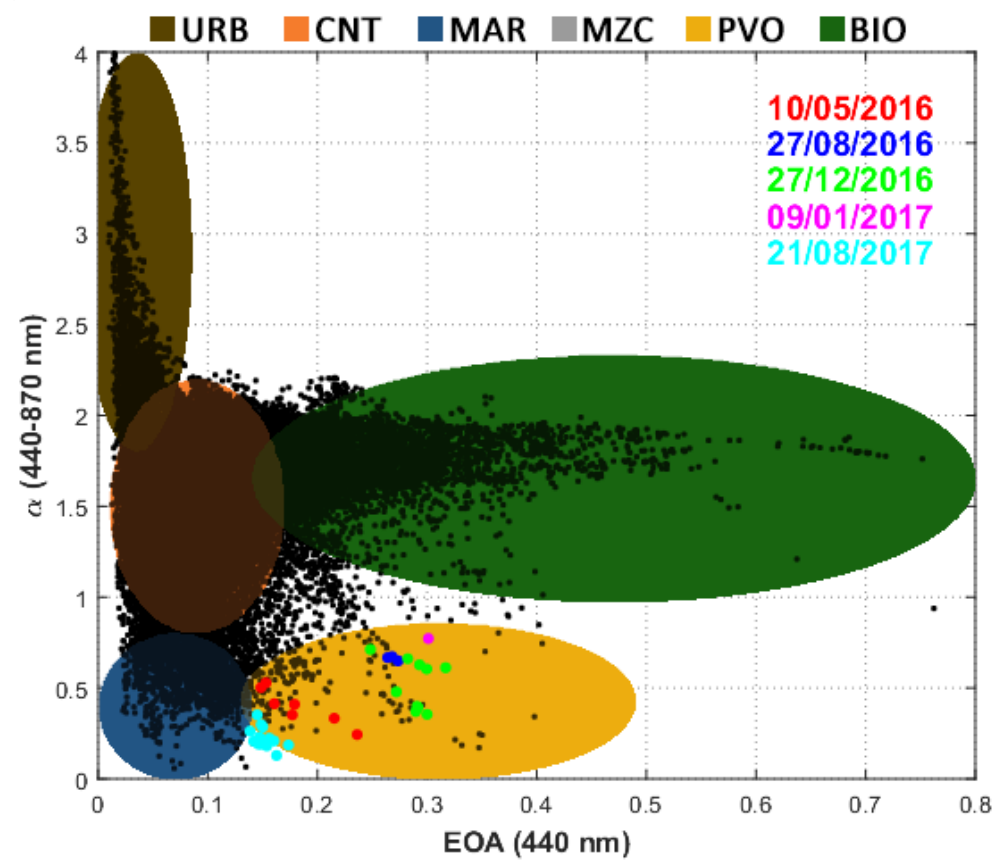

Fig. 1. Diagrama de dispersión entre el $\tau_{440} \mathrm{y} \alpha$. Se resaltan los eventos de polvo identificados como desérticos y sus fechas correspondientes.

Éste es un método comúnmente empleado para la clasificación de los aerosoles [15-19] y ha sido utilizado específicamente para la clasificación de aerosoles de tipo desértico en la Península Ibérica [20,21]. A partir de este diagrama y estableciendo rangos de valores, tanto para $\tau_{440}$ como para $\alpha$, se ha realizado la clasificación de los aerosoles, teniendo siempre en cuenta las condiciones locales y de entorno del sitio de medición. Se han clasificado los aerosoles en 6 tipos específicos: Urbanos (URB), Continentales (CNT), Marítimos (MAR), Mezcla (MZC), Biomasa (BIO) y Polvo (PVO). A partir de esta clasificación se encontraron, para el Nivel 1.5 un total de 985 horarios con aerosoles del tipo polvo mientras que para el Nivel 2.0 fueron un total de 175 horarios, casi 6 veces menos horarios. El valor medio del EOA para los aerosoles tipo polvo del Nivel 1.5 es de 0.339 con una desviación estándar de 0.648 , prácticamente dos veces el valor de la media lo cual es una muestra de la gran dispersión de datos, con un gran número de valores atípicos llegando a alcanzar el valor máximo de EOA = 7.3. Por su parte, en el caso de los valores de EOA para el Nivel 2.0, el valor medio para todos los casos fue de $0.19 \pm 0.06$, lo que demuestra mayor consistencia de la muestra. 
Teniendo en cuenta estos resultados y que uno de los aspectos que se tienen en cuenta para que los datos de EOA de AERONET alcancen el Nivel 2.0 es la consistencia de los datos, es que se decide emplear los datos de EOA correspondientes al Nivel 2.0. Todos los horarios con mediciones de aerosoles de tipo polvo fueron analizados para determinar si tienen o no, origen desértico.

La versión en línea del modelo de trayectorias HYSPLIT [22], se empleó para establecer las retrotrayectorias vinculadas con cada uno de los casos de polvo. Estas retrotrayectorias tuvieron su origen en el sitio de medición donde se encuentra instalado el fotómetro y se ejecutaron para un lapso de 72 horas hacia atrás, en tres niveles de altura: 500, 1500 y 3000 metros sobre el nivel del suelo. Los datos meteorológicos empleados para las ejecuciones del modelo fueron los del Sistema de Asimilación de Datos Globales (GDAS, por sus siglas en inglés) con una resolución de 0.5 grados.

Se consideraron como posibles eventos de origen desértico, aquellos casos en los que, al menos, una de las trayectorias cruce, al nivel del suelo, alguno de los desiertos costeros de Perú, área sombreada de color amarillo en la Fig. 2 (panel izquierdo), a una altura suficiente sobre la superficie como para que pueda arrastrar las partículas que han sido suspendidas por los vientos que se producen en la costa.
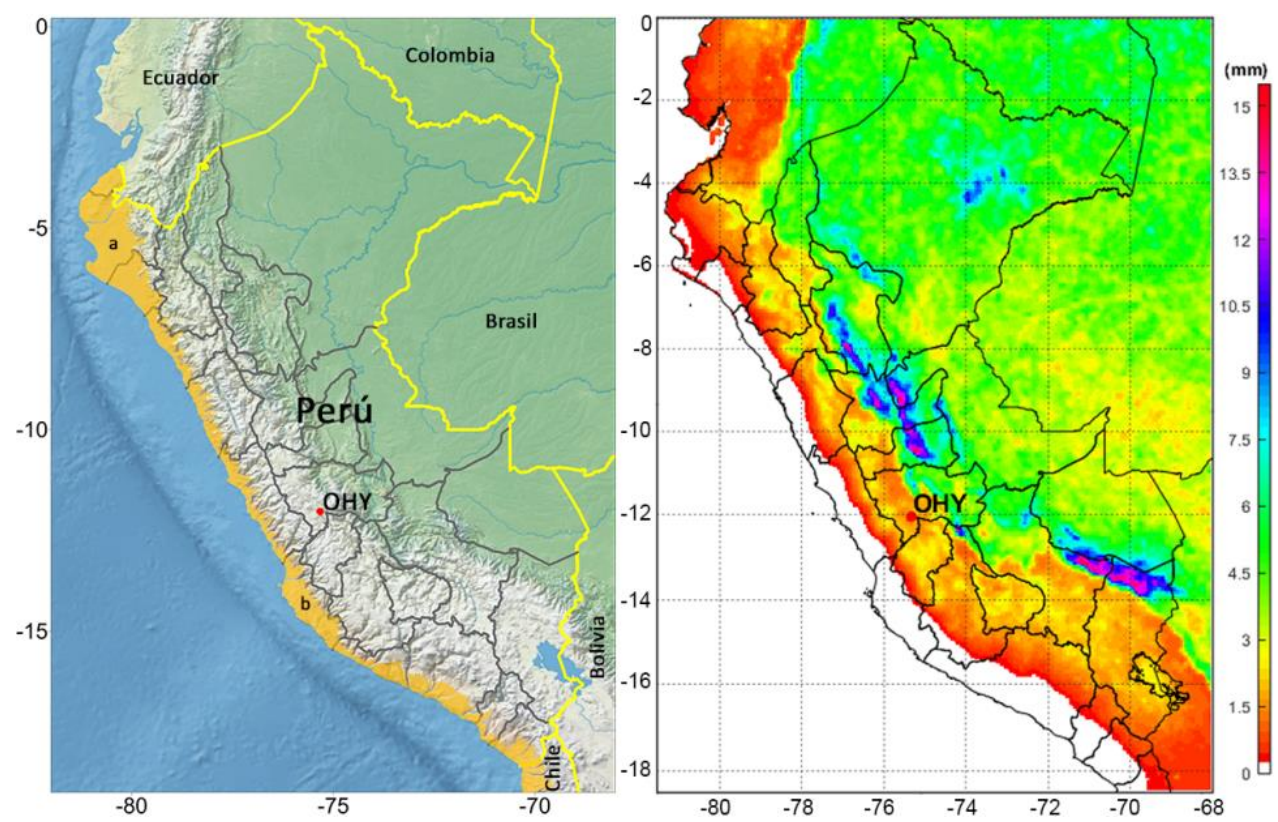

Fig. 2. Desierto Costero del Perú (sombreado en amarillo), en el panel izquierdo, donde se resaltan el desierto de Sechura (a), el de mayor extensión del país y el desierto de Ica (b), uno de los más conocidos. Se señala además la ubicación del Observatorio de Huancayo (OHY).

Como se aprecia en la figura, el desierto costero de Perú se encuentra ubicado entre el océano pacífico y la cordillera de los Andes, la cual constituye una barrera natural para el transporte de humedad desde el Amazonas. Por esta razón, la costa de Perú es una de las regiones más áridas del mundo, con escasas precipitaciones durante todo el año, como se observa en la Fig. 2 (panel derecho), donde se han graficado las precipitaciones medias para el período 1981-2013 [23] y donde se aprecia claramente la escases total de precipitaciones a lo largo de toda la costa peruana.

Se ha empleado el modelo de Pronóstico e Investigación del Tiempo (WRF, por sus siglas en inglés), con el objetivo de analizar el comportamiento de los vientos en las regiones de interés, para los casos que se consideren como eventos de polvo desértico.

\section{Resultados}

En la Fig. 3 se representan todas las mediciones de $\tau_{440}$ realizadas por el fotómetro solar ubicado en el OHY para el período de estudio y se resaltan, en puntos amarillos, los casos identificados como aerosoles del tipo polvo. De los 175 casos identificados como aerosoles de polvo, 23 (13\%) corresponden al año 2015, mientras que 83 (47 \%) corresponden al año 2016 y finalmente 69 (39 \%) al año 2017. Estos casos se 
resumen en 6 días del año 2015 con el $16 \%$ de los casos, 19 días (51 \%) corresponden al año 2016 y en el caso de 2017 un total de 12 días (32\%), con aerosoles del tipo polvo. El año 2017, a pesar de contar con un mes menos de datos que el año 2015, cuenta con el doble de días con mediciones de aerosoles de tipo polvo y con tres veces la cantidad de casos identificados.

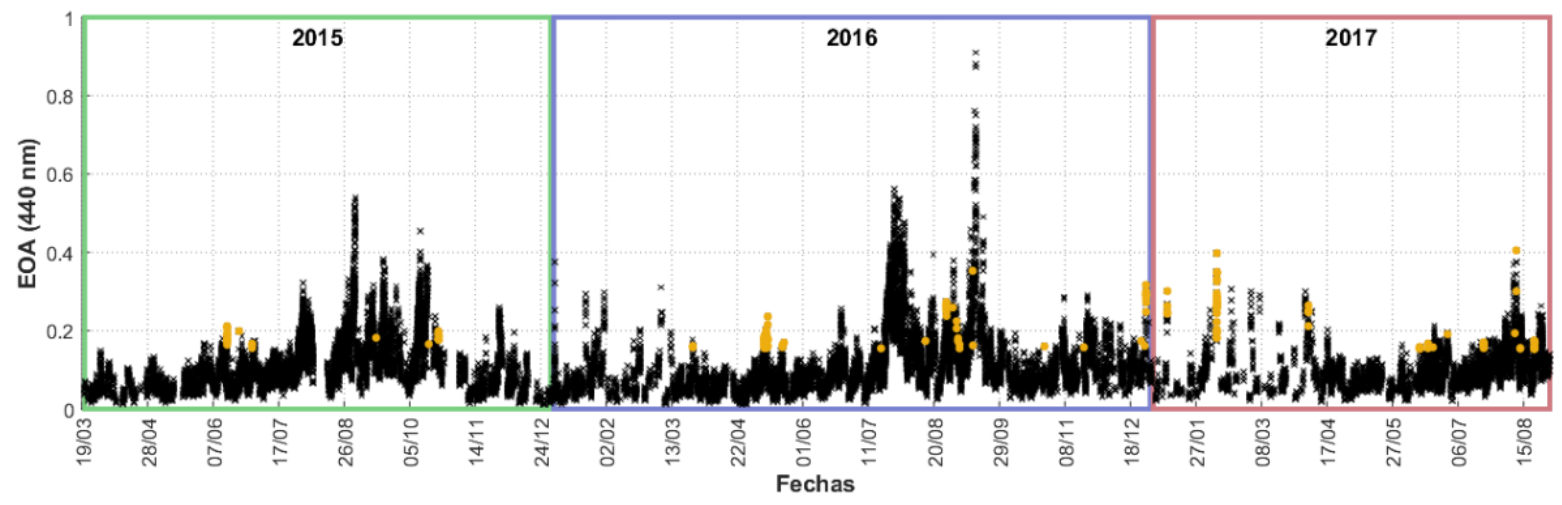

Fig. 3. Mediciones de Espesor Óptico por Aerosoles en la longitud de onda de $440 \mathrm{~nm}$ para el período de estudio. Se resaltan en amarillo los casos de aerosoles de tipo polvo.

A partir de las salidas del modelo HYSPLIT, para cada uno de los casos identificados como aerosoles de tipo polvo, se pudo determinar que de los 37 días con aerosoles del tipo polvo, solamente 5 días están asociados a polvo de origen desértico. Al año 2016 corresponden 3 días, mientras que al 2017 le corresponden solamente 2 días, para el 2015 no se encontró ningún día vinculado a eventos de polvo desértico. En la TABLA 1 se muestran las fechas y horarios de los días identificados como eventos de polvo desértico, aparecen también el número de mediciones tomadas en cuenta para el cálculo de los valores medios de $\tau_{440}$ y $\alpha$, así como, se indican los niveles correspondientes a las alturas de 500, 1500 y 3000 m, que cruzaron sobre alguno de los desiertos costeros de Perú. Los valores medios tanto de $\tau_{440}$ como de $\alpha$, fueron calculados a partir de las mediciones que se encontraron en torno a la hora en punto. Esto se hace debido a que para las ejecuciones del modelo HYSPLIT se consideran solamente horas exactas. No obstante, para el análisis de los eventos se tuvieron en cuenta cada una de las mediciones realizadas por el fotómetro solar e identificadas como aerosoles de tipo polvo. En la Fig. 1 estas mediciones se representan con puntos coloreados y en la columna $\mathrm{N}$ de la TABLA 1 se muestra la cantidad de mediciones tomadas en cuenta para el cálculo de las medias de $\tau_{440} \mathrm{y} \alpha$ para cada una de la Horas (GMT).

TABLA 1. Fechas y horarios de los días con eventos de polvo desértico y los niveles de las trayectorias que cruzaron sobre alguna de las regiones del desierto costero de Perú.

\begin{tabular}{|c|c|c|c|c|c|c|c|}
\hline \hline Fecha & Horas (GMT) & $\mathrm{N}$ & $\tau_{440}$ & $\alpha$ & $500 \mathrm{~m}$ & $1500 \mathrm{~m}$ & $3000 \mathrm{~m}$ \\
\hline \hline $10-05-2016$ & $14: 00,15: 00$ & 2,2 & $0.226,0.178$ & $0.289,0.381$ & $\mathrm{x}$ & $\mathrm{x}$ & \\
$27-08-2016$ & $12: 00,13: 00$ & 3,3 & $0.259,0.268$ & $0.755,0.664$ & $\mathrm{x}$ & & \\
$27-12-2016$ & $12: 00,13: 00$ & 2,2 & $0.317,0.282$ & $0.612,0.661$ & $\mathrm{x}$ & & \\
& $14: 00,15: 00$ & 1,5 & $0.248,0.289$ & $0.248,0.400$ & $\mathrm{x}$ & $\mathrm{x}$ & \\
$09-01-2017$ & $14: 00$ & 1 & 0.301 & 0.072 & & $\mathrm{x}$ & \\
$21-08-2017$ & $14: 00$ & 5 & 0.158 & 0.216 & & & $\mathrm{x}$ \\
\hline \hline
\end{tabular}

Como se puede observar en la tabla anterior, los cuatro horarios vinculados con eventos de polvo desértico se encuentran entre las 12:00 y las 15:00 GMT, o sea, entre las 7:00 y las 10:00 hora local (HL), con predominio del horario de las 14:00 GMT, o sea, a las 9:00 HL con el $45.5 \%$ de los horarios. Llama la atención el hecho de que, en el año 2016, en todas las fechas seleccionadas existen como mínimo dos horarios consecutivos con eventos de polvo de origen desértico, llegando a un máximo de 4 horarios consecutivos con estos eventos el día 27-12-2016. Por el contrario, en las dos fechas que corresponden al año 2017, los eventos de polvo ocurren en un solo horario, coincidiendo en ambos casos con las 14:00 GMT.

Se puede apreciar igualmente que en ninguna de las fechas seleccionadas coincidieron las tres trayectorias, por el contrario, predominan con el $75 \%$ de los horarios presentados, los casos con una sola trayectoria. Solamente existen dos fechas (10-05-2016 y 27-12-2016) en las cuales coinciden dos niveles de trayectorias (500 y 1500 metros), en ambos casos, en los horarios de las 14:00 y las 15:00 GMT. De todas las fechas de 
eventos relacionados con polvo desértico, existe solo un caso vinculado a la trayectoria del nivel de 3000 metros la cual es, además, el único nivel coincidente.

El máximo valor medio $\left(\tau_{440}=0.317\right)$ se registró el 27-12-2016 en el horario de las 12:00 GMT y corresponde a la trayectoria de $500 \mathrm{~m}$, este máximo estuvo seguido por el valor de $\tau_{440}=0.301$ registrado el día 09-01-2017 a las 14:00 GMT en la trayectoria de $1500 \mathrm{~m}$. De acuerdo a estudios realizados sobre los vientos Paracas, empleando trampas de arena, el mayor aporte de masa de partículas finas de polvo desértico tiene lugar durante estos meses [1]. El mínimo valor medio $\left(\tau_{44} 0=0.158\right)$ se registró el día 21-082017, en el horario de las 14:00 GMT, en la trayectoria de $3000 \mathrm{~m}$. El resto de los valores de $\tau_{440}$ oscilan entre 0.178 y 0.289 .

Existen varios casos, fuera de las fechas seleccionadas, en los cuales la trayectoria de 3000 metros cruza por encima de alguno de los desiertos costeros. Algunos coinciden incluso con avisos de fuertes vientos en la costa emitidos por el Servicio Nacional de Meteorología e Hidrología del Perú (SENAMHI). Sin embargo, estos casos no han sido considerados debido a que las trayectorias, al momento de cruzar por encima de los desiertos, lo hacen a alturas superiores a los $3000 \mathrm{~m}$, por lo que no es posible que arrastren los aerosoles que se generan en superficie como consecuencia de vientos fuertes en estas regiones. Igual sucede, pero en mucho menor cuantía, con casos en las trayectorias de 500 y $1500 \mathrm{~m}$, que al pasar sobre los desiertos costeros lo hacen a alturas superiores a los $2000 \mathrm{~m}$.

La gran mayoría de los casos (88 \%) que han sido identificados como aerosoles de tipo polvo a partir de las mediciones del fotómetro solar, y que no han sido clasificados como desérticos, se encuentran asociados a otras fuentes. Una de estas fuentes, y de hecho la que se considera como la principal generadora de los eventos de polvo que afectan el OHY está relacionada con la preparación de tierras para la agricultura. Esto debido a que el $\mathrm{OHY}$ se encuentra ubicado en medio de tierras dedicadas al cultivo. Otra posible fuente entre los años 2015 y 2016, fue la construcción de una carretera en las cercanías del observatorio, donde se generó gran cantidad de partículas que fueron liberadas a la atmósfera. Si se realiza un análisis de los casos u horarios de aerosoles del tipo polvo, por épocas del año, la mayor contribución tiene lugar en el período poco lluvioso con el $61 \%$ de todos los casos. La mayor contribución en este periodo tiene lugar durante el otoño (marzo a mayo) con el $31 \%$ mientras que el invierno (junio a agosto) contribuye con el $30 \%$. Durante el verano (diciembre a febrero) tiene lugar el $28 \%$ de los casos, mientras que en la primavera se alcanza apenas el $10 \%$ de los casos, lo que evidencia que durante el período lluvioso los mecanismos de deposición húmeda provocan que exista una menor concentración de aerosoles, en general, y de polvo en particular.

Es importante señalar que para ninguna de las fechas seleccionadas como eventos de polvo desértico existen productos de inversión de AERONET. Esto impide que puedan realizarse análisis de las propiedades ópticas de estos aerosoles, así como, de la distribución volumétrica de tamaños de partículas que permitan caracterizar los aerosoles de origen desértico [24]. Los aerosoles de polvo junto a los de origen marítimo se consideran aerosoles de gran tamaño en comparación con otros tipos de aerosoles y conforman la fracción gruesa de la distribución de tamaños. Con el objetivo de determinar si los aerosoles medidos durante los eventos de polvo analizados corresponden a partículas gruesas y, por tanto, relacionados con aerosoles de polvo de origen desértico, se han empleado datos de la fracción fina y gruesa del EOA, información derivada del Algoritmo de Deconvolución Espectral (ADE) [25,26]. A continuación, se analizarán de forma individual las fechas seleccionadas como eventos de polvo desértico y finalmente, se analizará el comportamiento de las fracciones finas y gruesas de EOA para los casos identificados como desérticos.

\section{3.a Evento del 10-05-2016}

En la Fig. 4 se representan las retrotrayectorias (panel izquierdo) correspondientes al 10 de mayo de 2016 para los horarios de las 14:00 (líneas continuas) y las 15:00 GMT (líneas discontinuas). Para este día tanto la trayectoria de $500 \mathrm{~m}$ como la de $1500 \mathrm{~m}$ cruzan el desierto costero al nivel del suelo (Fig. 4, panel derecho superior), arrastrando así las partículas de polvo suspendidas en la atmósfera. Aproximadamente 24 horas transcurrieron, para ambos niveles de altura, desde que las trayectorias cruzaron sobre el desierto costero hasta que arribaron al OHY. Los tres niveles de trayectorias, en ambos horarios, tienen su origen sobre el océano y todas cruzan el desierto costero en el departamento de Lima, en una franja no mayor a $84 \mathrm{~km}$. Las simulaciones de viento en superficie realizadas con el modelo WRF (Fig. 4, panel derecho inferior), para la fecha y los horarios correspondientes a este evento, muestran que efectivamente existe un giro de los vientos, que tienen una componente aproximadamente al norte noroeste (NNO) sobre el océano, hacia el este noreste (ENE), justo en toda la región comprendida entre el norte (N) del departamento de Ica y sur (S) del departamento de Lima. Frente a esta zona costera se aprecia sobre el mar una franja a lo largo de la 
costa con vientos cuyas velocidades se encuentran entre los 4 y los $5 \mathrm{~m} / \mathrm{s}$, los cuales deben haber dado lugar al levantamiento de las partículas de polvo que luego fueron arrastradas hasta el OHY.
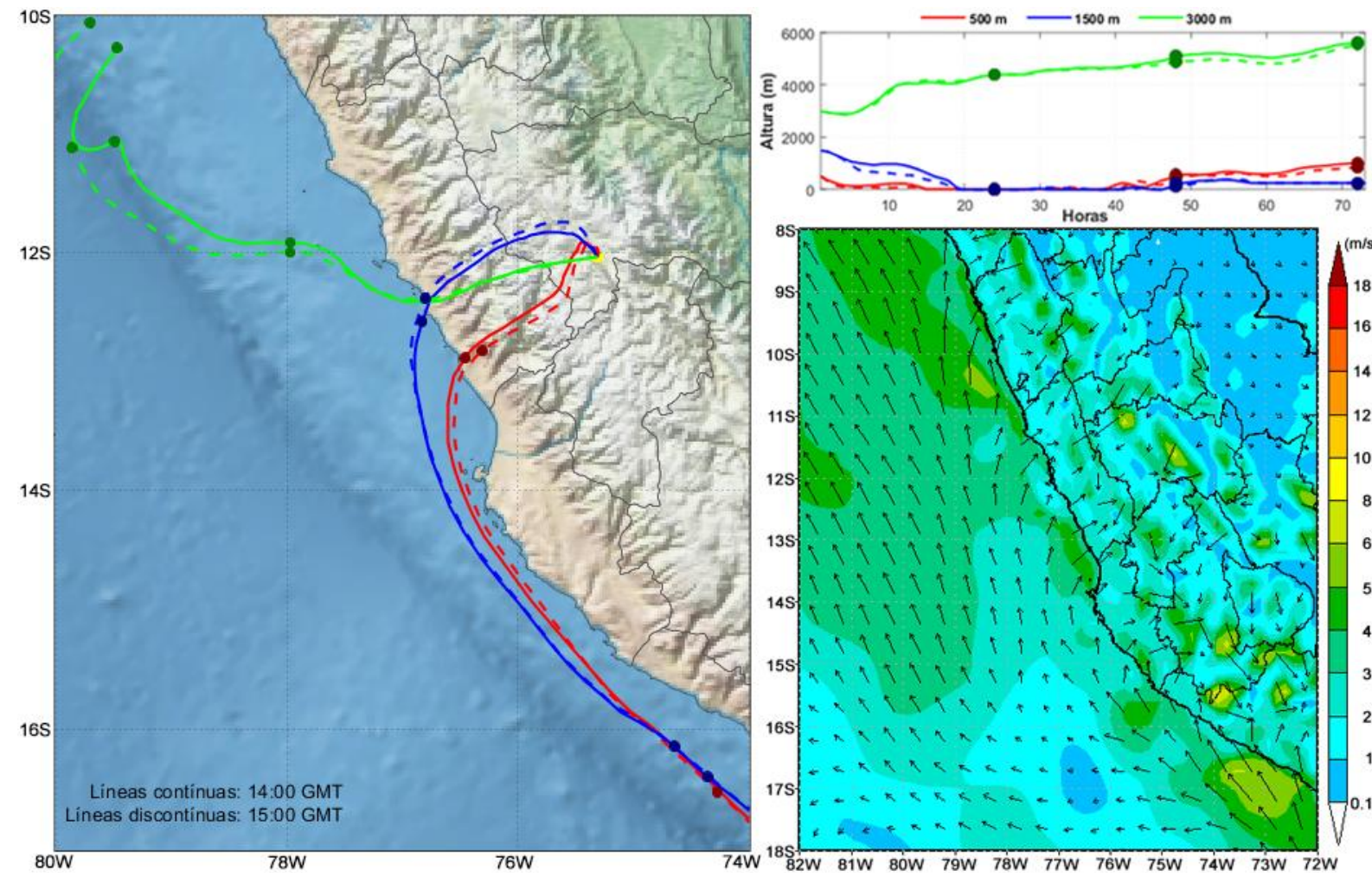

Fig. 4. Evento correspondiente al 10 de mayo de 2016 en los horarios de las 14:00 y las 15:00 GMT. Se muestran las retrotrayectorias en los niveles de 500,1500 y 3000 metros (panel izquierdo), al igual que la distribución vertical de las trayectorias (panel derecho superior) para todos los horarios identificados. La simulación del modelo WRF, para la fecha y horarios identificados, se muestra en el panel derecho inferior.

De acuerdo con las mediciones de espesor óptico por aerosoles realizadas en esta fecha, por el fotómetro solar ubicado en el $\mathrm{OHY}$, fueron dos los tipos de aerosoles registrados además del tipo desértico, estos fueron los tipos continentales y marítimos. Un total de 41 mediciones de $\tau_{440}$ se registraron en el Nivel 2.0 para esta fecha, de las cuales solamente 5 corresponden a aerosoles de tipo desérticos, lo que representa el $12 \%$, de todos los casos; por su parte, 11 mediciones corresponden a aerosoles marítimos (27\%), mientras que la mayoría de las mediciones, 25 en total, corresponden al tipo continental con el $61 \%$ de los casos. Si se considera la carga de aerosoles para este día como la suma de los espesores ópticos medios de los tres tipos de aerosoles registrados $\left(\tau_{440}=0.389\right)$, se tendría que el mayor aporte corresponde, sin embargo, a los aerosoles de tipo desérticos con $\tau_{440}=0.194$, lo que constituiría un aporte del $50 \%$ a la carga de aerosoles para esta fecha. En el caso de los aerosoles de tipo marítimo el espesor óptico es de 0.115 (29\%), mientras que en último lugar se encuentra el tipo continental con apenas un valor de 0.081 (21\%).

\section{3.b Evento del 27-08-2016}

Las retrotrayectorias que corresponden al 27 de agosto de 2016, para los horarios de las 12:00 y las 13:00 GMT se muestran en el panel izquierdo de la Fig. 5. Con líneas continuas se representan las trayectorias del horario de las 12:00 GMT mientras que en líneas discontinuas se muestran las trayectorias de las 13:00 GMT. En esta fecha solamente la trayectoria de $500 \mathrm{~m}$ cruza el desierto costero, por el departamento de Lima, próximo a la ciudad de Lima, para ambos horarios. Al igual que en el caso anterior, aproximadamente 24 horas les tomó a ambas trayectorias llegar al OHY, luego de cruzar sobre el desierto. Estas dos trayectorias, a diferencia de las de 1500 y $3000 \mathrm{~m}$, que se encuentran todo el tiempo sobre el continente, tiene su origen y transcurre durante las 72 horas sobre el océano. Todo el tiempo, hasta aproximadamente 17 horas antes de llegar al OHY, las dos trayectorias de $500 \mathrm{~m}$ viajan sobre el nivel del mar y de la superficie terrestre, como se puede apreciar en la parte superior del panel derecho de la Fig. 5. La simulación realizada con el WRF, que se muestra en la parte inferior del panel derecho de esta figura, muestra un contraste bien marcado en cuanto a las velocidades y la dirección del viento sobre el océano y el continente. Sobre el continente predominan vientos inferiores a los $2 \mathrm{~m} / \mathrm{s}$ sin una dirección bien definida, mientras que sobre 
el océano los vientos tienen velocidades por encima a los $8 \mathrm{~m} / \mathrm{s}$ se mueven prácticamente paralelos a la línea de costa hasta sobrepasar la Península de Paracas cuando realizan un leve giro con rumbo al NNE, coincidiendo así con lo que muestran las trayectorias. Ya sobre el continente los vientos giran más al este lo que hace posible que la trayectoria alcance el OHY en menos de 24 horas, arrastrando consigo las partículas de polvo suspendidas sobre la región.
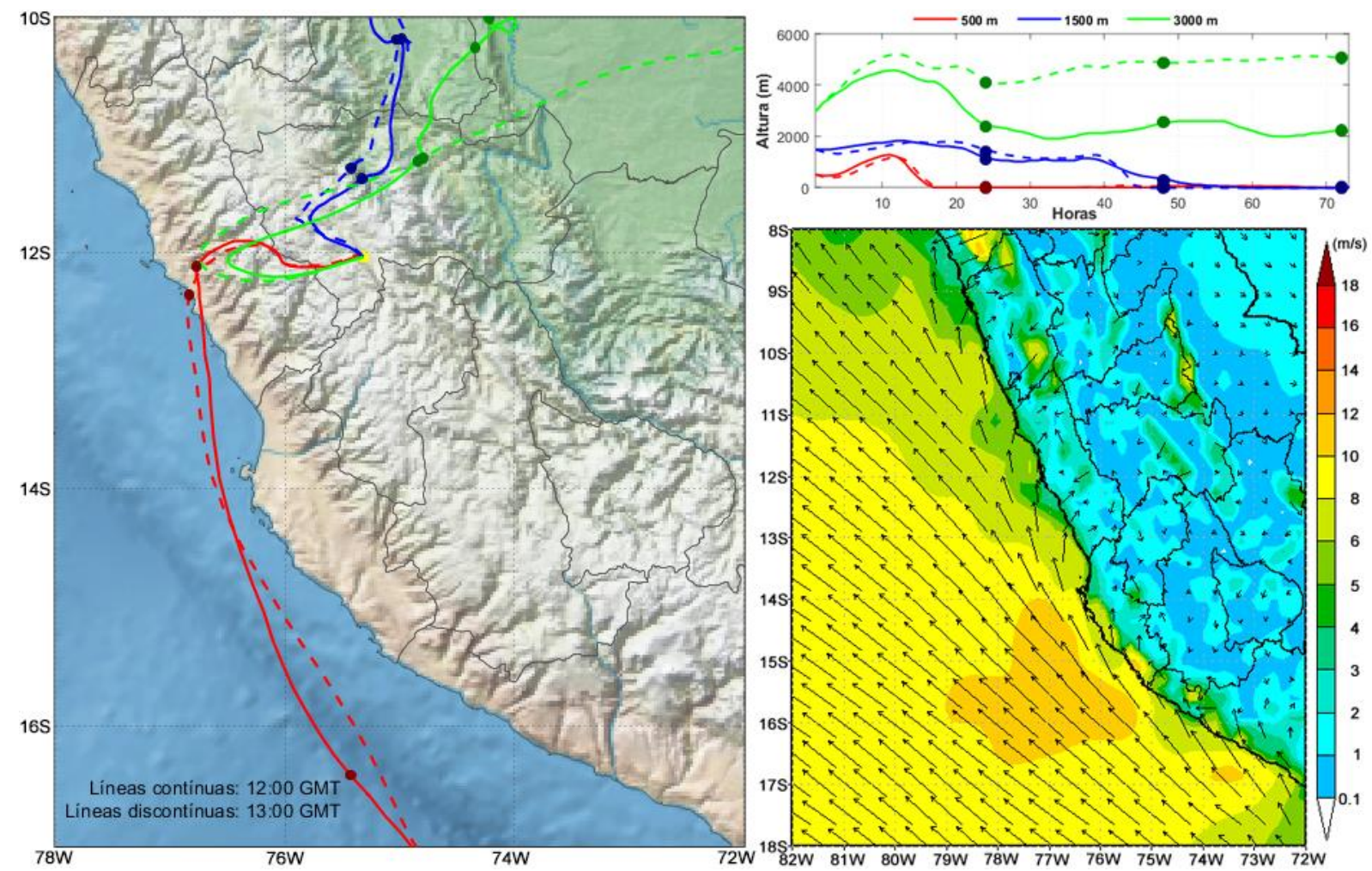

Fig. 5. Evento correspondiente al 27 de agosto de 2016 en los horarios de las 12:00 y las 13:00 GMT. Se muestran las

retrotrayectorias en los niveles de 500, 1500 y 3000 metros (panel izquierdo), al igual que la distribución vertical de las trayectorias (panel derecho superior) para todos los horarios identificados. La simulación del modelo WRF, para la fecha y horarios identificados, se muestra en el panel derecho inferior.

En cuanto a la composición de los aerosoles registrados por el fotómetro solar para esta fecha, además del tipo desértico, que constituye el $20 \%$ del total de mediciones realizadas (41) este día, se encuentran aerosoles de tipo biomasa (44\%), mezcla (34\%) y finalmente con apenas el $2 \%$, el tipo de aerosol continental. A pesar de que la trayectoria de $500 \mathrm{~m}$ tiene su origen y transcurre prácticamente todo el tiempo sobre el océano, no se registran para esta fecha aerosoles de tipo marítimo. Si al igual que en el caso anterior, se considerara la carga de aerosoles total como la suma de las medias $\left(\tau_{440}=0.797\right)$ de las mediciones de espesor óptico de cada uno de los tipos de aerosoles, se obtendría nuevamente que el mayor aporte lo realizan los aerosoles de tipo desértico con el $33 \%\left(\tau_{440}=0.260\right)$, seguido del tipo mezcla con el $26 \%\left(\tau_{440}=0.208\right)$, el tipo biomasa con el $22 \%\left(\tau_{440}=0.179\right)$ y finalmente el tipo continental con un espesor óptico medio de 0.151 para el $19 \%$.

\section{3.c Evento del 27-12-2016}

La fecha del 27 de diciembre de 2016 tiene la particularidad de que cuenta con cuatro horarios en los cuales se han identificado eventos de aerosoles de tipo desértico. En dos de estos horarios (12:00 y 13:00 GMT) se registró una sola trayectoria $(500 \mathrm{~m})$ cruzando sobre el desierto costero y en los otros dos horarios (14:00 y 15:00 GMT), son dos las trayectorias (500 y $1500 \mathrm{~m}$ ) que cruzan sobre estos desiertos. Debido a que las trayectorias de $500 \mathrm{~m}$ son muy similares en los cuatro horarios y a que las simulaciones realizadas con el modelo WRF son prácticamente las mismas para los cuatro horarios, se muestra solamente la Fig. 6 correspondiente a los horarios de las 14:00 y las 15:00 GMT.

La trayectoria de $500 \mathrm{~m}$, en los cuatro horarios mencionados, tiene su origen sobre el océano pacífico a unos $300 \mathrm{~km}$ frente a la costa de Iquique, Chile, y cruzan el desierto costero peruano en torno a la frontera entre 
los departamentos de Lima e Ica. A diferencia del resto de los horarios, la trayectoria de $500 \mathrm{~m}$ de las 14:00 GMT pasa sobre el desierto de Paracas antes de cruzar definitivamente el desierto costero entre los departamentos antes mencionados. La región comprendida entre las ciudades de Pisco y San Juan en el sur peruano, dentro de la cual se encuentra el desierto de Paracas, es ampliamente conocida por la intensidad de los vientos en superficie que allí se generan [27,28]. Estos vientos se conocen comúnmente como "Vientos Paracas", los cuales, combinados con las amplias zonas desérticas que allí se encuentran, dan lugar a grandes tormentas de polvo [1] con una frecuencia de 4 a 5 eventos por año como promedio [13,14].

Todas las trayectorias del nivel de $500 \mathrm{~m}$ viajan al nivel de la superficie, hasta que luego de cruzar el desierto se encuentran con la sierra de los Andes y comienzan a ascender. Como promedio, 24 horas toman en llegar estas trayectorias al OHY una vez cruzan sobre el desierto, excepto la trayectoria de las 15:00 GMT que llega a tardar unas 40 horas. En el caso de la Fig. 6 (panel izquierdo) se observa que las trayectorias de $1500 \mathrm{~m}$, para los horarios de las 14:00 y las 15 GMT, se originan en el océano e ingresan al continente por la porción más occidental del departamento de Arequipa, tomando aproximadamente unas 42 horas en alcanzar el OHY. El flujo de los vientos sobre el océano, con dirección noroeste (NO) y paralelos a la costa, con velocidades entre 4 y $8 \mathrm{~m} / \mathrm{s}$ se puede apreciar en la simulación realizada con el WRF (panel derecho inferior) de la figura. Igualmente se puede observar como ingresa el viento al continente tanto en la porción occidental de Arequipa como por la región ubicada en las fronteras entre del departamento de Ica y Lima, coincidiendo con lo representado en las trayectorias.
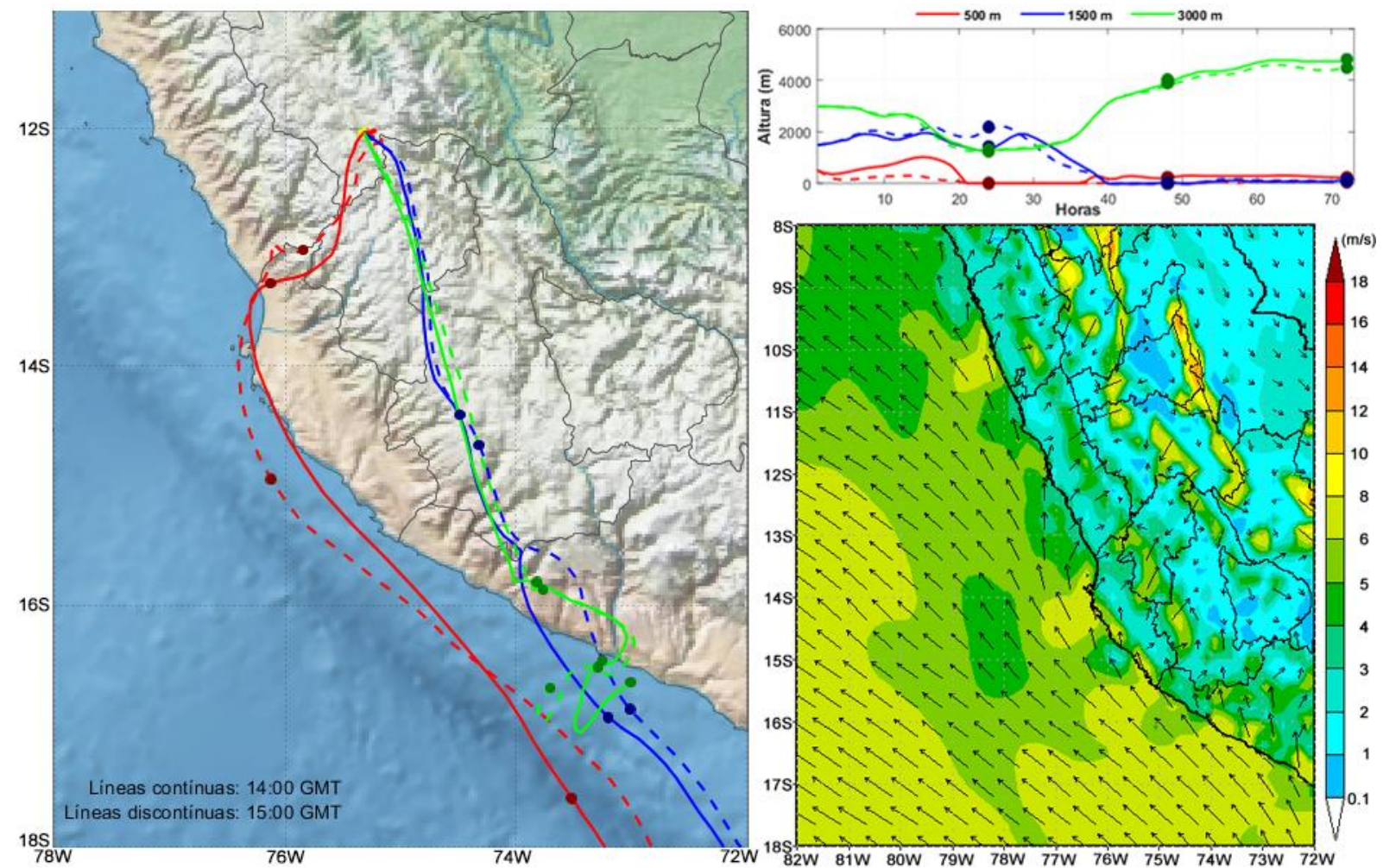

Fig. 6. Evento correspondiente al 27 de diciembre de 2016 en los horarios de las 14:00 y las 15:00 GMT. Se muestran las retrotrayectorias en los niveles de 500, 1500 y 3000 metros (panel izquierdo), al igual que la distribución vertical de las trayectorias (panel derecho superior) para todos los horarios identificados. La simulación del modelo WRF, para la fecha y horarios identificados, se muestra en el panel derecho inferior.

Un total de 23 mediciones realizadas por el fotómetro se reportan para esta fecha, de las cuales 10 corresponden a aerosoles desérticos (43\%), siendo de todos los eventos de polvo desértico seleccionados, el que cuenta con mayor número de mediciones. Los tipos mezcla y continental son los otros dos tipos de aerosoles que se registraron en esta fecha con un total de 8 (35\%) y 5 (22\%) casos, respectivamente. Los porcientos de contribución al espesor óptico de cada uno de los tipos, de acuerdo a los criterios más arriba considerados, son muy similares a los porcientos anteriores. El mayor espesor óptico lo aporta el tipo desértico $\left(\tau_{440}=0.288\right)$, seguido del tipo mezcla $\left(\tau_{440}=0.212\right)$ y en último lugar el continental $\left(\tau_{440}=0.130\right)$.

3.d Eventos del 09-01-2017 y 21-08-2017 
En el año 2017 solo se identificaron dos fechas con aerosoles de tipo desértico, el 9 de enero y el 21 de agosto y en ambos casos, se ha identificado una sola trayectoria y un solo horario, a diferencia de los eventos del 2016, donde, como mínimo, se identificaron siempre dos horarios con eventos de polvo desértico. El evento del día 9 de enero tiene un comportamiento muy similar a los ya descritos hasta ahora por lo que no será abordado en detalles. La trayectoria identificada en este caso corresponde a la de 1500 m, cruzando el desierto costero por una región ubicada al sur del departamento de Ica. Esta fecha fue la de menor número de mediciones de aerosoles del tipo polvo con apenas 4, siendo el $24 \%$ de todas las mediciones para este día (17). El mayor número de mediciones correspondió al tipo biomasa con un total de 10 (59\%) mientras que del tipo mezcla se registraron solamente 3 mediciones (18\%). Sin embargo, los porcientos de aporte, al espesor óptico por aerosoles para este día, son muy similares para los tres tipos (desértico 37 \%, biomasa $30 \%$ y mezcla $30 \%$ ), imponiéndose, mínimamente, el tipo desértico.

La fecha del 21 de agosto (Fig. 7) es el único caso, de todos los identificados en este trabajo, en el que la trayectoria de los $3000 \mathrm{~m}$ es la que aporta los aerosoles de tipo desértico al OHY, siendo además la única trayectoria que cruza sobre el desierto costero para este día. Esta trayectoria tiene su origen en el océano (Fig. 7, panel izquierdo) y atraviesa todo el desierto de Ica, uno de los mayores de Perú después del desierto de Sechura al norte del país, con rumbo norte noroeste (NNO). Esta trayectoria transcurre todo el tiempo al nivel de la superficie (Fig. 7, panel derecho superior) hasta llegar a los Andes donde comienza su ascenso hasta llegar a los $3000 \mathrm{~m}$ sobre el OHY. Desde que esta trayectoria ingresa al continente hasta que arriba al OHY, transcurren aproximadamente un total de 64 horas, debido al lazo que describe esta trayectoria, el cual tiene su explicación al analizar la simulación realizada con el WRF (Fig. 7, panel derecho inferior). A diferencia de los casos anteriormente analizados, sobre el continente, específicamente sobre la sierra de los Andes, existe un flujo orientado desde la sierra hacia la costa, con velocidades superiores a los $16 \mathrm{~m} / \mathrm{s}$ en algunas regiones de la sierra central. En este caso el viento sobre el océano no tiene un flujo exactamente paralelo a la costa, incluso, frente a la costa central existe una región con velocidades inferiores a los $3 \mathrm{~m} / \mathrm{s}$, justo frente a la zona de vientos fuertes de la sierra central. Sin embargo, en la región por donde ingresa la trayectoria de $3000 \mathrm{~m}$ los vientos en la costa llegan a ser superiores a los $4 \mathrm{~m} / \mathrm{s}$. Una vez sobre el continente el flujo de viento asociado a esta trayectoria sufre el embate de los vientos que bajan de la sierra, cambiando el sentido del flujo, lo que provoca finalmente la formación del lazo que describe esta trayectoria.
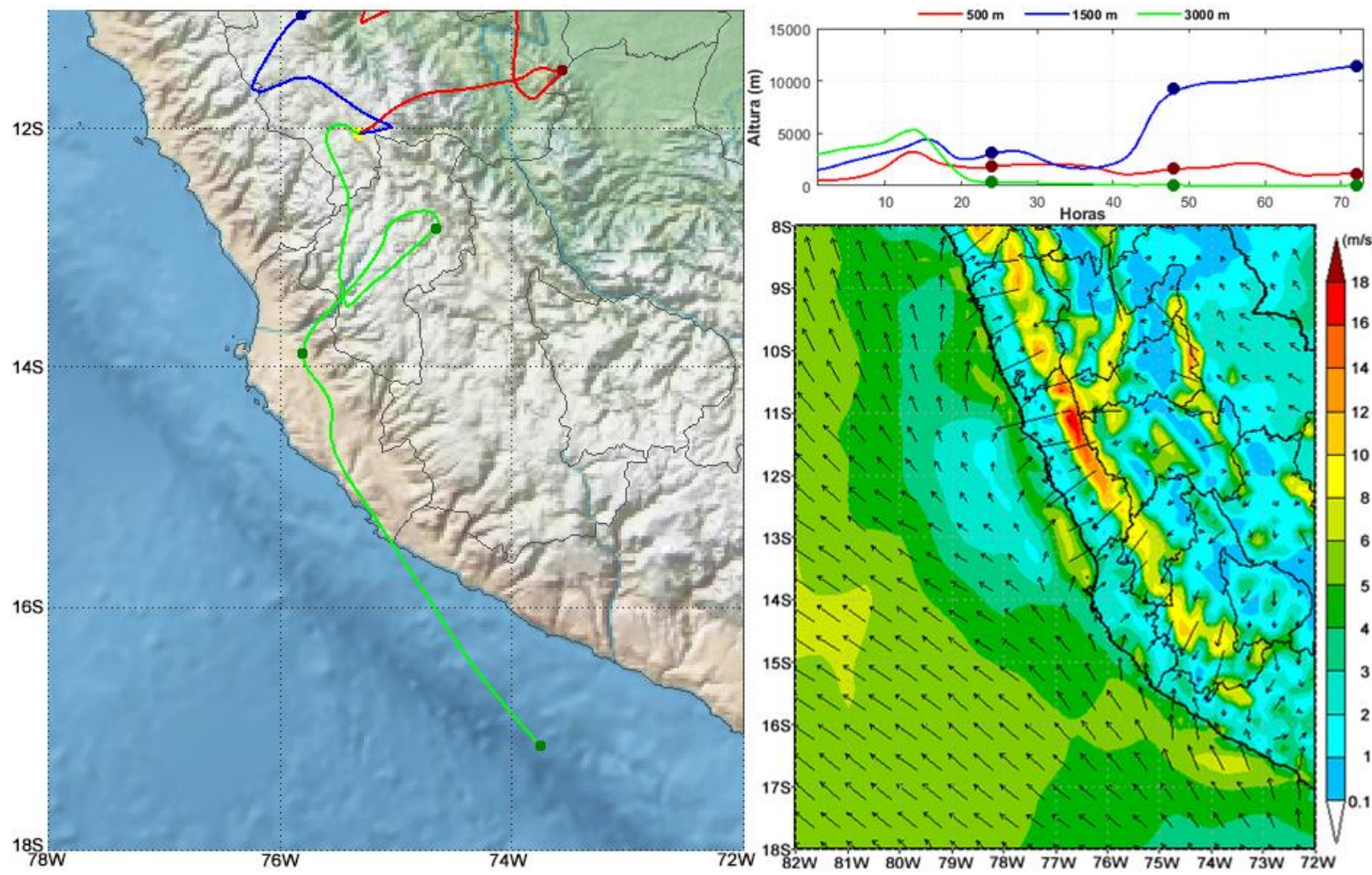

Fig. 7. Evento correspondiente al 21 de agosto de 2017 en el horario de niveles de 500, 1500 y 3000 metros (panel izquierdo), al igual que la distribución vertical de las trayectorias (panel derecho superior) para todos los horarios identificados. La simulación del modelo WRF, para la fecha y horarios identificados, se muestra en el panel derecho inferior. 
Para esta fecha un total de 114 mediciones registradas por el fotómetro solar se encuentran en el Nivel 2.0 de AERONET, de ellas solamente 8 corresponden a los aerosoles del tipo desértico lo que representa a penas, el $7 \%$ de los casos. El tipo continental llega a 49 registros con el $43 \%$ mientras que el tipo marítimo alcanza los 57 registros con el $50 \%$ de los casos. Sin embargo, el mayor aporte al espesor óptico $\left(\tau_{440}=0.386\right)$ para este día lo tiene el tipo desértico con el $42 \%\left(\tau_{440}=0.163\right)$ seguido del tipo marítimo con el $32 \%$ y finalmente el tipo continental con el $25 \%$.

Como se ha podido apreciar, en todos los casos identificados como eventos de polvo desértico, la mayor contribución al espesor óptico por aerosoles lo ha tenido siempre el tipo de aerosol clasificado como desértico. Esto demuestra la importancia de la búsqueda de estos eventos dentro de los casos identificados como polvo en las mediciones realizadas con el fotómetro solar ubicado en el OHY.

$\mathrm{Al}$ analizar el campo de la presión atmosférica no ha sido posible identificar, dado el pequeño número de casos, entre otras razones, los patrones que describan las condiciones sinópticas que propician el arrastre de los aerosoles desérticos hacia la sierra central. Por otro lado, el gradiente de presión es en general débil, por lo que el predominio de determinadas condiciones locales en la circulación, provocan que, fundamentalmente en los horarios comprendidos entre las 12:00 y las 15:00 GMT, sean transportados estos aerosoles desde los desiertos costeros hacia el Valle del Mantaro.

\section{3.e Análisis de las fracciones finas y gruesas del EOA}

Los valores de las fracciones finas y gruesas del EOA, derivados a partir del algoritmo de deconvolución espectral, para todos los horarios dentro de las cinco fechas seleccionadas como eventos de polvo desértico, se muestran en la Fig. 8. Los días considerados como eventos de aerosoles desérticos en el año 2016 (10 de mayo, 27 de agosto y 27 de diciembre), se muestran en los gráficos a, b y c, respectivamente, mientras que las fechas del año 2017 ( 9 de enero y 21 de agosto), se muestran en los gráficos d y e, respectivamente. Las áreas sombreadas en cada una de las gráficas corresponden a la duración del evento identificado como desértico, conteniendo los puntos (horarios) considerados dentro del evento. En los ejes de las abscisas se representan los horarios de medición en horas GMT.

De acuerdo con la clasificación de aerosoles mostrada en epígrafes anteriores, la fecha del 10 de mayo de 2016 (Fig. 8 a), es la única fecha para la cual se registra la presencia de aerosoles de tipo MAR además de los aerosoles de tipo PVO, considerados como desérticos en los horarios entorno a las 14 y las 15 GMT. Por esta razón podría considerarse para esta fecha, principalmente para el horario de las 15 GMT, la existencia de una mezcla de aerosoles de polvo desértico con aerosoles de tipo MAR. Puede apreciarse que, sobre todo para el entorno de las 14 GMT, las diferencias en magnitud entre el modo fino y el grueso son considerables. Esto, aparejado al hecho de que el valor del Parámetro de Angstrom $(\alpha=0.289)$ es muy pequeño para este horario (TABLA 1), lo que significa la presencia de partículas muy grandes, consistentes con el tipo polvo y que los aerosoles de tipo marítimo se registran a partir del horario de las 15 GMT, podría considerarse que en este horario predominan los aerosoles desérticos.

En la (Fig. 8 b) se muestran los modos finos y gruesos del EOA derivados del ADE para el 27 de agosto de 2016. En este caso los eventos identificados como aerosoles desérticos corresponden a los horarios de las 12 y las 13 GMT. En ambos casos el modo grueso se encuentra por encima del modo fino, sin embargo, las diferencias entre ambos modos son superiores en el horario de las 13 GMT. El resto de mediciones registradas en esta fecha están relacionados con aerosoles de tipo BIO, es por ello que en el resto de los horarios el modo fino predomina sobre el modo grueso, excepto en los últimos horarios donde se registran aerosoles de tipo PVO, pero no relacionados con los desiertos costeros. La fecha del 27 de diciembre de 2016 (Fig. 8 c), es la única fecha donde se han identificado 4 horarios con aerosoles desérticos. En todos los eventos identificados como aerosoles desérticos el modo grueso se encuentra por encima del modo fino, resaltando los relacionados con el horario de las 15 GMT donde la diferencia entre ambos modos es superior al resto de los horarios. Durante este día se clasificaron, además de los aerosoles de polvo, los tipos BIO y CNT, estos dos tipos de aerosoles son mucho más pequeños que los de polvo y como se puede observar, los valores del modo fino, derivados del ADS, son superiores al modo grueso.

Las fechas identificadas en el año 2017 con aerosoles desérticos corresponden al 9 de enero (Fig. 8 d) y al 21 de agosto (Fig. 8 e). En ambos casos solamente se registra un evento con este tipo de aerosol y en el caso del mes de enero, sólo contempla un horario de medición vinculado al evento. Previo a las 14 GMT (horario del evento), existen algunos casos de aerosoles de tipo polvo, pero no vinculados a ninguno de los desiertos costeros del Perú. En el resto de los horarios de esta fecha los aerosoles están relacionados con el tipo BIO 
y como se puede apreciar, el modo fino predomina sobre el modo grueso para el resto de los horarios. La fecha del 21 de agosto de 2017 es un caso particular en el sentido de que existe una gran cantidad de horarios con aerosoles de PVO, el 57 \% de las mediciones de este día son de tipo PVO mientras que el $43 \%$ corresponden al tipo CNT. Luego del análisis de cada uno de los horarios con aerosoles del tipo PVO se determinó que las mediciones registradas en torno a las 14 GMT son la únicas vinculadas con aerosoles desérticos. En el resto de los casos las fuentes generadoras de polvo parecen estar relacionadas con áreas más próximas al OHY de acuerdo con las trayectorias analizadas.
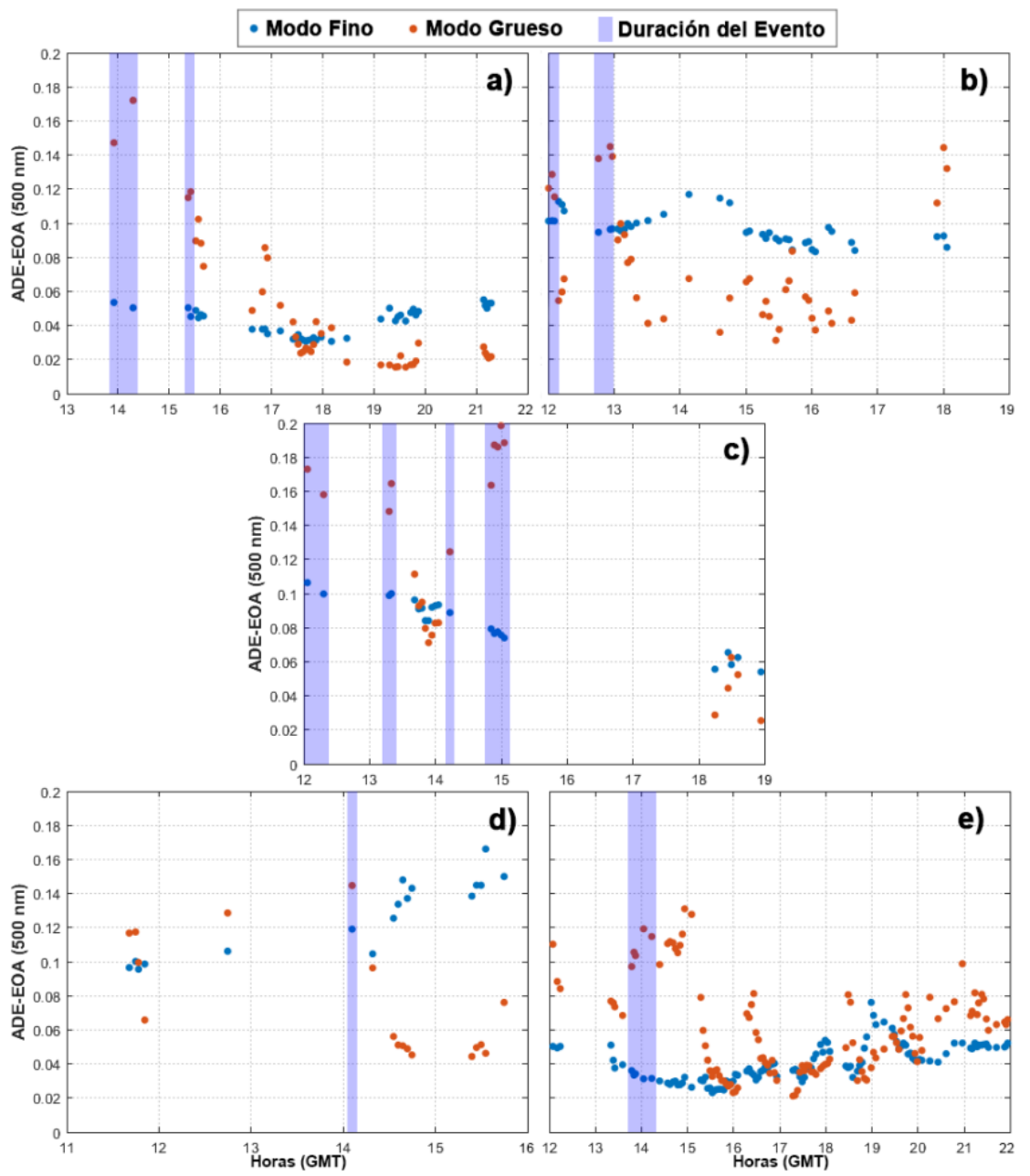

Fig. 8. Fracciones finas y gruesas del EOA, derivados a partir del ADE para las fechas de 10-05-2016 (a), 27-08-2016 (b), 27-12-2016

(c), 09-01-2017 (d) y 21-08-2017 (e). Las zonas sombreadas representan la duración de cada evento identificado como desértico.

\section{Conclusiones}

Este trabajo constituye la primera evidencia para el Perú, del transporte de aerosoles de origen desértico hacia los Andes centrales, específicamente hacia el Valle del Mantaro, donde se encuentra ubicado el Observatorio de Huancayo. De un total de 175 mediciones de aerosoles identificados como tipo polvo, solamente 21 casos pudieron ser considerados como polvo de origen desértico, lo que representa a penas, el $12 \%$ de todos los casos. Estos casos se concentran en 37 días de mediciones con aerosoles de tipo polvo de los cuales solamente en 5 de ellos se encontró evidencia de un origen desértico. Debido a la falta de información sobre las propiedades ópticas y de otros parámetros no fue posible hacer una caracterización de los mismos. Se emplearon, sin embargo, los datos generados por el algoritmo de deconvolución espectral del espesor óptico por aerosoles, lo que permitió caracterizar, para cada evento, las relaciones entre los modos finos y gruesos del EOA. Este análisis permitió corroborar la presencia y predominio de partículas gruesas en los eventos identificados como desérticos. El empleo conjunto de retrotrayectorias y de simulaciones del modelo WRF, permitió corroborar el transporte de estas partículas desde los desiertos costeros hacia el interior de Perú. Dada la dificultad que entraña, a partir de las mediciones disponibles, la determinación del origen desértico de los aerosoles considerados como tal en este estudio se emplearán, en 
lo posible, para futuros trabajos, otras técnicas de medición que permitan una mejor caracterización de los aerosoles vinculados a los desiertos del Perú.

\section{Agradecimientos}

Este trabajo se desarrolló dentro del proyecto "Estudio de la variación de las propiedades ópticas de los aerosoles debido a las quemas de vegetación para evaluar su influencia en el forzamiento radiativo terrestre", financiado por CONCYTEC, bajo el contrato: 010-2013-FONDECYT. Partes de este trabajo fueron presentadas en el "IX Workshop on Lidar Measurements in Latinamerica, July 17-22, 2016, Santos, Brazil". 